\title{
Zeros of Networks of Linear Multi-Agent Systems: Time-Invariant Interconnections
}

\author{
Mohsen Zamani* \\ Research School of Engineering \\ Australian National University \\ Canberra, ACT 0200, Australia \\ mohsen.zamanidanu. edu. au \\ Uwe Helmke** \\ Institute of Mathematics \\ University of Würzburg \\ 97074 Würzburg, Germany \\ Brian D.O. Anderson*** \\ Research School of Engineering \\ Australian National University \\ Canberra, ACT 0200, Australia \\ helmke@mathematik. uni-wuerzburg. de brian.anderson@anu.edu.au
}

\begin{abstract}
This paper investigates the zero properties of networks of linear multi-agent control systems, where the coupling parameters between the agents are assumed to be constant. We characterize the zeros both for heterogeneous and homogeneous networks. Moreover, for homogenous networks with time-invariant interconnection dynamics and SISO agents, we illustrate how zeros of each individual agent and zeros of interconnection dynamics contribute to the zero properties of the whole network. We also investigate the effects of blocking on the zeros.
\end{abstract}

\section{INTRODUCTION}

The importance of zeros for the analysis and design of linear control systems has been recognized in system engineering for a very long time. Early work in this direction is due to Rosenbrock [19] who emphasized the relevance of multivariable zeros in systems theory. Zeros provide obstructions to the existence of inverses of linear systems and the zero dynamics of a system are important in adaptive control and high gain feedback analysis. In this paper, we examine the zero properties of networks of linear dynamical systems that arise through static interconnections of a finite number of linear control systems. Such networks occur naturally in modelling multi-agent linear systems, with applications to e.g. cyclic pursuit [14]; shortening flows in image processing [4] or for the discretization of partial differential equations [3]. It is also worthwhile mentioning that analysis of networks of multi-agent systems has recently absorbed even more attentions, due to its importance in cyber-physical security [15] and [20] ${ }^{1}$.

Dynamics of networks of multi-agent systems have been extensively studied in the literature [17], [16]. For instance, in [16] the stability properties of a network of agents interconnected by the consensus law are explored. However, the zero properties of a network of multi-agent systems have not been studied so far and require a more detailed

\footnotetext{
*Support by ARC Discovery Project Grant DP1092571 is gratefully acknowledged.

**Support by the grant HE 1858/12-2 from the German Research Foundation within the DFG SPP 1305 program is gratefully acknowledged.

***This work is supported by Australian Research Council's Discovery Projects DP-0877562 and DP-110100538. The author is also supported by NICTA (National ICT Ltd. Australia)

${ }^{1}$ More details on this will be provided in the extended version of this paper prepared for journal submission.
}

study; this paper is a step in that direction.

An important step for the future planned extension of our analysis to periodic, or more generally time-varying, interconnection parameters is to study blocking or lifting techniques for networks with time-invariant interconnections. This topic is also addressed in this paper. Note that blocking of linear time-invariant systems is useful in the multirate sampled-data systems controller design as shown by [5] and [13]. Furthermore, from a theoretical perspective, the pole properties of the blocked systems are well understood, see e.g. [1], [13], whereas much less is known about the zero properties of the blocked systems. References [2], [11], [23] and [6] have analysed the zero properties of blocked systems obtained from blocking of time-invariant systems. The results in those references show under restrictive assumptions that the blocked system has a zero if and only if its associated linear time-invariant unblocked system has a zero. The assumptions in [23], [6] on the normal rank and the structure of the transfer function matrices were subsequently relaxed by [22]. Proofs provided in the above references are lengthy and do not take any underlying network structure consideration. In the last part of this paper, we extend these results for arbitrary heterogeneous networks of linear systems using a much simpler proof idea based on circulant-like matrices. This technique provides additional insight into the problem, enables us to deal with the system matrix directly and almost certainly should help in tackling the zero properties of systems with periodic topology.

The structure of this paper is as follows. First, in Section II we introduce state-space and higher order polynomial system models for time-invariant networks of linear control systems. A central result stated here is the strict system equivalence between these different system representations. Section III characterizes completely the finite and infinite zeros of arbitrary heterogeneous networks. For homogeneous networks of identical SISO systems more explicit results are shown. Homogeneous networks with a circulant coupling topology are studied as well. In Section IV first a relation between the transfer function of blocked systems and the transfer function of the associated unblocked systems is recalled. We then relate the zeros of blocked networked control systems to the zeros of their corresponding unblocked 
systems. Finally, Section V provides the concluding remarks.

\section{Models of Interconnected Systems}

We consider networks of $N$ linear multi-agent systems, coupled through constant interconnection parameters. Each agent is assumed to have the state-space representation of a linear discrete-time control systems

$$
\begin{aligned}
x_{i}(t+1) & =A_{i} x_{i}(t)+B_{i} v_{i}(t) \\
w_{i}(t) & =C_{i} x_{i}(t), i=1, \ldots, N .
\end{aligned}
$$

Here $A_{i} \in \mathbb{R}^{n_{i} \times n_{i}}, B_{i} \in \mathbb{R}^{n_{i} \times m_{i}}$ and $C_{i} \in \mathbb{R}^{p_{i} \times n_{i}}$ are the associated system matrices. We assume that each system is controllable and observable. We assume that each agent is interconnected by static coupling laws as

$$
v_{i}(t)=\sum_{j=1}^{N} L_{i j} w_{j}(t)+R_{i} u(t) \in \mathbb{R}^{m_{i}}
$$

with $L_{i j} \in \mathbb{R}^{m_{i} \times p_{j}}$ and $R_{i} \in \mathbb{R}^{m_{i} \times m}$ and $u(t)$ an external input to the whole network. Further, there is an interconnected output given by

$y(t)=\sum_{i=1}^{N} S_{i} w_{i}(t)+D u(t)$ with $S_{i} \in \mathbb{R}^{p \times p_{i}}, i=1, \ldots, N$.

Define $\bar{m}=\sum_{i=1}^{N} m_{i}, \bar{p}=\sum_{i=1}^{N} p_{i}, \bar{n}=\sum_{i=1}^{N} n_{i}$ and matrices as

$$
\begin{aligned}
& L=\left(L_{i j}\right)_{i j} \in \mathbb{R}^{\bar{m} \times \bar{p}} \quad R=\left(\begin{array}{c}
R_{1} \\
\vdots \\
R_{N}
\end{array}\right) \in \mathbb{R}^{\bar{n} \times m} \\
& S=\left(S_{1}, \ldots, S_{N}\right) \in \mathbb{R}^{p \times \bar{n}} \quad D \in \mathbb{R}^{p \times m}
\end{aligned}
$$

and

$$
\begin{aligned}
& A=\operatorname{diag}\left(A_{1}, \ldots, A_{N}\right), \quad B=\operatorname{diag}\left(B_{1}, \ldots, B_{N}\right) \\
& C=\operatorname{diag}\left(C_{1}, \ldots, C_{N}\right), \quad x(t):=\left(\begin{array}{c}
x_{1}(t) \\
\vdots \\
x_{N}(t)
\end{array}\right) \in \mathbb{R}^{\bar{n}} .
\end{aligned}
$$

Then the closed-loop system is

$$
\begin{aligned}
x(t+1) & =\mathbf{A} x(t)+\mathbf{B} u(t) \\
y(t) & =\mathbf{C} x(t)+D u(t),
\end{aligned}
$$

with matrices

$$
\mathbf{A}:=A+B L C \quad \mathbf{B}:=B R, \quad \mathbf{C}:=S C .
$$

One can also start by assuming that system (1) is defined in terms of Rosenbrock-type equations [18] i.e. by systems of higher order difference equations

$$
\begin{aligned}
T_{i}(\sigma) \xi_{i} & =U_{i}(\sigma) v_{i} \\
w_{i} & =V_{i}(\sigma) \xi_{i} .
\end{aligned}
$$

Here $\sigma$ denotes the backwards shift operator that acts on sequences of vectors $(\xi(t))_{t}$ as $(\sigma \xi)(t)=\xi(t-1)$. Furthermore, $T_{i}, U_{i}, V_{i}$ denote polynomial matrices of sizes $T_{i}(z) \in \mathbb{R}^{r_{i} \times r_{i}}, U_{i}(z) \in \mathbb{R}^{r_{i} \times m_{i}}$ and $V_{i}(z) \in \mathbb{R}^{p_{i} \times r_{i}}$, respectively. We always assume that $T_{i}(z)$ is nonsingular, i.e. that $\operatorname{det} T_{i}(z)$ is not the zero polynomial. Moreover, system (4) is assumed to be strictly proper, i.e. we assume that the associated transfer function

$$
G_{i}(z)=V_{i}(z) T_{i}(z)^{-1} U_{i}(z)
$$

is strictly proper. Following Fuhrmann [10], any strictly proper system (4) of higher order difference equations has an associated state-space realization $(A, B, C)$, the so-called shift realization, such that the polynomial matrices

$$
\Sigma(z)=\left(\begin{array}{cc}
z I-A & -B \\
C & 0
\end{array}\right), \quad \Pi(z)=\left(\begin{array}{cc}
T(z) & -U(z) \\
V(z) & 0
\end{array}\right)
$$

are strict system equivalent [10]. If a first order representation (1) is strict system equivalent to the higher order system (4) then of course the associated transfer functions coincide, i.e. we have

$$
C_{i}\left(z I-A_{i}\right)^{-1} B_{i}=V_{i}(z) T_{i}(z)^{-1} U_{i}(z) .
$$

Throughout this paper we assume that the first order and higher order representations i.e. systems (1) and (4), are chosen to be of minimal order, respectively. This is equivalent to the controllability and observability of the shift realizations (1) associated with these representations (4). It is also equivalent to the simultaneous left coprimeness of $T_{i}(z), U_{i}(z)$ and the right coprimeness of $T_{i}(z), V_{i}(z)$. Proceeding as above, define polynomial matrices

$$
T(z)=\operatorname{diag}\left(T_{1}(z), \ldots, T_{N}(z)\right) \in \mathbb{R}[z]^{\bar{r} \times \bar{r}}
$$

and similarly for $V(z)$ and $U(z)$. Here $\bar{r}=\sum_{i=1}^{N} r_{i}$. Using this notation, we write all $N$ systems of (4) in the matrix form as

$$
\left(\begin{array}{c}
0 \\
I
\end{array}\right) w=\left(\begin{array}{cc}
T(\sigma) & -U(\sigma) \\
V(\sigma) & 0
\end{array}\right)\left(\begin{array}{l}
\xi \\
v
\end{array}\right) .
$$

Then we have the left- and right coprime factorizations of the node transfer function as

$$
G(z)=C(z I-A)^{-1} B=V(z) T(z)^{-1} U(z) .
$$

The interconnections are given, as before, by

$$
\begin{aligned}
& v=L w+R u \\
& y=S w+D u .
\end{aligned}
$$

The resulting network representation then becomes

$$
\left(\begin{array}{c}
0 \\
I
\end{array}\right) y=\left(\begin{array}{cc}
T(\sigma)-U(\sigma) L V(\sigma) & -U(\sigma) R \\
S V(\sigma) & D
\end{array}\right)\left(\begin{array}{l}
\xi \\
u
\end{array}\right)
$$

with network transfer function defined as

$$
\begin{aligned}
\Gamma(z) & =\mathbf{C}(z I-\mathbf{A})^{-1} \mathbf{B}+D \\
& =S V(z)(T(z)-U(z) L V(z))^{-1} U(z) R+D
\end{aligned}
$$

The connection between the state-space and polynomial matrix representations (2) and (10), respectively, is clarified by the following recent result. This theorem implies that important system-theoretic properties such as reachability 
and observability, as well as the poles and zeros of the network (2) of multi-agent systems can all be characterized by the polynomial system matrix (10).

Theorem 1 ([9]): The interconnected systems (2) and (10) are strict system equivalent. In particular, there exist unimodular matrices $\tilde{P}(z), \tilde{Q}(z)$ such that for each $q \geq \max (\bar{n}, \bar{r})$

$$
\begin{aligned}
& \tilde{P}(z)\left(\begin{array}{ccc}
I_{q-\bar{n}} & 0 & 0 \\
0 & z I-\mathbf{A} & -\mathbf{B} \\
0 & \mathbf{C} & D
\end{array}\right) \tilde{Q}(z)= \\
& \left(\begin{array}{ccc}
I_{q-\bar{r}} & 0 & 0 \\
0 & T(z)-U(z) L V(z) & -U(z) R \\
0 & S V(z) & D
\end{array}\right)
\end{aligned}
$$

\section{Zeros of Multi-Agent Networks}

As a consequence of Theorem 1 we derive a complete characterization for the zeros of network of systems (2). We first present an extension of the classical definition of zeros [12] to higher order systems. Note that the normal rank $\operatorname{grk} G(z)$ of a rational matrix function $G(z)$ is defined as

$$
\operatorname{grk} G(z)=\max \{\operatorname{rank} G(z) \mid z \in \mathbb{C}, G(z) \neq \infty\} .
$$

Definition 1: Let $U(z), V(z), T(z)$ be polynomial matrices with $T(z) \in \mathbb{R}[z]^{\bar{r} \times \bar{r}}$ nonsingular such that the $\bar{p} \times \bar{m}$ transfer function $V(z) T(z)^{-1} U(z)$ is strictly proper; let $D$ be a constant $\bar{p} \times \bar{m}$ matrix. A finite zero of a polynomial system matrix

$$
\bar{\Pi}(z)=\left(\begin{array}{cc}
T(z) & -U(z) \\
V(z) & D
\end{array}\right)
$$

is any complex number $z \in \mathbb{C}$ such that

$$
\operatorname{rank} \bar{\Pi}(z)<\operatorname{grk} \bar{\Pi}(z)
$$

holds. $\bar{\Pi}(z)$ is said to have a zero at infinity if

$$
\bar{r}+\operatorname{rank} D<\operatorname{grk} \bar{\Pi}(z) \text {. }
$$

As a consequence of Fuhrmann's result [10], a polynomial system matrix (12) has a finite or infinite zero if and only if the polynomial matrix

$$
\bar{\Sigma}(z)=\left(\begin{array}{cc}
z I-A & -B \\
C & D
\end{array}\right)
$$

of the associated shift realization $(A, B, C, D)$ has a finite or infinite zero. Theorem 1 thus leads to our first main result on zeros of interconnected systems; see [9] for a proof. We emphasize that the characterization of zeros in the subsequent Theorem 2 holds for any interconnection matrices and does not require any assumptions on reachability or observability of the network, except of those for the individual node systems.

Theorem 2: Consider the strictly proper node transfer function $G(z)$ with minimal representations

$$
G(z)=C(z I-A)^{-1} B=V(z) T(z)^{-1} U(z) .
$$

Let $L, R, S, D$ denote arbitrary constant interconnection matrices and let

$$
\Gamma(z)=S V(z)(T(z)-U(z) L V(z))^{-1} U(z) R+D
$$

denote the network transfer function. Then

1) For all $z \in \mathbb{C}$

$$
\begin{aligned}
& \operatorname{rank}\left(\begin{array}{cc}
z I-A-B L C & -B R \\
S C & D
\end{array}\right) \\
& =\bar{n}-\bar{r}+\operatorname{rank}\left(\begin{array}{cc}
T(z)-U(z) L V(z) & -U(z) R \\
S V(z) & D
\end{array}\right) .
\end{aligned}
$$

2) $(A+B L C, B R, S C, D)$ has a finite zero at $z \in \mathbb{C}$ if and only if

$$
\begin{aligned}
& \operatorname{rank}\left(\begin{array}{cc}
T(z)-U(z) L V(z) & -U(z) R \\
S V(z) & D
\end{array}\right)< \\
& \bar{r}+\operatorname{grk} \Gamma(z) .
\end{aligned}
$$

3) $(A+B L C, B R, S C, D)$ has a zero at infinity if and only if

$$
\operatorname{rank} D<\operatorname{grk} \Gamma(z) .
$$

In particular, if $D$ has full row rank or full column rank, then $(A+B L C, B R, S C, D)$ has no infinite zero.

\section{A. Homogeneous Networks}

The preceding result has a nice simplification in the case of homogeneous networks of SISO agents, i.e. where the node systems $\left(A_{i}, B_{i}, C_{i}\right)$ are identical SISO systems. Let us define the interconnection transfer function as

$$
\phi(z)=S(z I-L)^{-1} R+D ;
$$

note that in the SISO case $L_{i j}$ is a scalar; hence, $L$ is a square matrix.

Theorem 3: Assume that $\left(A_{i}, b_{i}, c_{i}\right)$ are identical SISO systems; let $p(z) / q(z)$ be a coprime polynomial factorization of the identical transfer functions $g(z)=c_{i}\left(z I-A_{i}\right)^{-1} b_{i}$ and define $h(z)=1 / g(z)$. Let $L, R, S, D$ denote any constant interconnection matrices.

1) The homogeneous network $(\mathbf{A}, \mathbf{B}, \mathbf{C}, D)$ has a finite zero at $z \in \mathbb{C}$ with $p(z) \neq 0$ if and only if $h(z) \in \mathbb{C}$ is a finite zero of $(L, R, S, D)$.

2) $(\mathbf{A}, \mathbf{B}, \mathbf{C}, D)$ has a zero at infinity if and only if $(L, R, S, D)$ has a zero at infinity.

3) $(\mathbf{A}, \mathbf{B}, \mathbf{C}, D)$ has a finite zero at $z \in \mathbb{C}$ with $p(z)=0$ if and only if $(L, R, S, D)$ has a zero at infinity.

Proof: Note that the network transfer function $\Gamma(z)=$ $D+p(z) S\left(q(z) I_{N}-p(z) L\right)^{-1} R$ has normal rank equal to the normal rank of $\phi(z)=D+S\left(z I_{N}-L\right)^{-1} R$, i.e. it coincides with the normal rank of $L, R, S, D$. By the preceding theorem, $z \in \mathbb{C}$ is a zero of $(\mathbf{A}, \mathbf{B}, \mathbf{C}, D)$ if and only if

$$
\operatorname{rank}\left(\begin{array}{cc}
q(z) I_{N}-p(z) L & -p(z) R \\
S & D
\end{array}\right)<N+\operatorname{grk} \phi(z) .
$$


For $p(z) \neq 0$ this is equivalent to

$$
\operatorname{rank}\left(\begin{array}{cc}
h(z) I_{N}-L & -R \\
S & D
\end{array}\right)<N+\operatorname{grk} \phi(z),
$$

i.e., to $h(z)$ being a finite zero of $(L, R, S, D)$. The statement of the second item follows easily from item 3 in Theorem 2 and the fact that $\Gamma(z)$ and $\phi(z)$ have the same normal rank. Now, we prove item $3 ; z \in \mathbb{C}$ is a zero of $(\mathbf{A}, \mathbf{B}, \mathbf{C}, D)$ if and only if inequality (13) holds. If $p(z)=0$, then by coprimeness we have $q(z) \neq 0$ and therefore we can restate (13) as

$$
\operatorname{rank}\left(\begin{array}{cc}
q(z) I_{N} & 0 \\
S & D
\end{array}\right)<N+\operatorname{grk} \phi(z) .
$$

This implies that $\operatorname{rank} D<\operatorname{grk} \phi(z)$. Thus a zero of the node transfer function $g(z)$ is a zero of $(\mathbf{A}, \mathbf{B}, \mathbf{C}, D)$ if and only if $(L, R, S, D)$ has a zero at infinity. This completes the proof.

Now assume that $D$ has full column rank or full row rank. Then the homogeneous network realization $(\mathbf{A}, \mathbf{B}, \mathbf{C}, D)$ has no zero at infinity. Thus in this case the finite zeros of $(\mathbf{A}, \mathbf{B}, \mathbf{C}, D)$ are exactly the preimages of the finite zeros of $(L, R, S, D)$ under the rational function $h(z)$. We conclude with a result that is useful for the design of networks with prescribed zero properties. The result below has a certain similarity with a result in Fax and Murray [8]. As shown by them, a formation of $N$ identical vehicles can be analysed for stability by analysing a single vehicle with the same dynamics modify by only a scalar, which assumes values equal to the eigenvalues of the interconnection matrix. Such a result is to do with poles, linking those of the individual agent and the overall system via the eigenvalues (which are pole-like) of the interconnection matrix. Our result is to do with zeros, but still links those of the individual agent, those of the interconnection matrix (suitably interpreted) and those of the whole system.

Recall, that a strictly proper real rational transfer function $g(z)$ is called lossless [21] if all poles of $g(z)$ are in the open unit disc and $|g(z)|=1$ holds for all $|z|=1$. A key property used below is that $|g(z)|>1$ if $|z|<1$ and $|g(z)|<1$ if $|z|>1$.

Corollary 1: Assume that $D$ has full column rank or full row rank. Then

1) The homogeneous network $(\mathbf{A}, \mathbf{B}, \mathbf{C}, D)$ has no zeros at infinity. A complex number $z$ is a finite zero of $(\mathbf{A}, \mathbf{B}, \mathbf{C}, D)$ if and only if $h(z) \neq \infty$ is a finite zero of $L, R, S, D$.

2) Assume that the agent transfer function $g(z)$ is lossless. Then $(\mathbf{A}, \mathbf{B}, \mathbf{C}, D)$ is a minimum phase network, i.e. all of its zeros have absolute value $<1$, if and only if $(L, R, S, D)$ is minimum phase.

Proof: The first claim is an immediate consequence of Theorem 3. If $g(z)$ is lossless then $|g(z)|<1$ holds if and and only if $|z|>1$. Thus $h(z)=1 / g(z)$ maps the complement of the open unit disc onto itself. Thus $|z| \geq 1$ if and only if $|h(z)| \geq 1$. Therefore $(L, R, S, D)$ has a finite zero $w$ with $|w| \geq 1$ if and only if each $z$ with $h(z)=w$ satisfies $|z| \geq 1$ and is a zero of $(\mathbf{A}, \mathbf{B}, \mathbf{C}, D)$. Note that for any finite $w$, there is necessarily a $z$ satisfying $h(z)=w$, since this is a polynomial equation for $z$. This proves the result.

We now extend the second part of the above corollary for the choice of passive transfer functions [21]. Let us recall that $g(z)$ is passive if and only if

1) all poles of $g(z)$ are in $|z| \leq 1$

2) $|g(z)| \leq 1 \quad \forall|z|=1$

and it is further true that

1) $|g(z)|<1 \quad \forall|z|>1$

2) If $|g(z)|>1$, then $|z|<1$.

Corollary 2: Assume that $D$ has full column rank or full row rank and $g(z)$ is passive. Then $(\mathbf{A}, \mathbf{B}, \mathbf{C}, D)$ is a minimum phase network, i.e. all of its zeros have absolute value $<1$, if $(L, R, S, D)$ is minimum phase.

Proof: Suppose $|z|$ is a finite zero of $\{A, B, C, D\}$. Then $h(z)$ is a finite zero of $\{L, S, R, D\}$. Then $1 / g(z)$ is a finite zero of $\{L, S, R, D\}$. By the minimum phase assumption, $|(1 / g(z))|<1$ or $|g(z)|>1$. By the observation above applying to passive transfer functions, $|z|<1$.

\section{B. Design of Networks}

An important issue is the construction of network topologies so that the resulting multi-agent network is zero-free, i.e. it does not have any finite zeros (but still may have a zero at infinity). We derive a simple sufficient condition for homogeneous networks. By Corollary 1, a homogeneous network $(\mathbf{A}, \mathbf{B}, \mathbf{C}, D)$ is zero-free if and only if $(L, R, S, D)$ is zero-free. For simplicity, we assume $m=p=1$ and $D=0$. Thus the interconnection transfer function $\phi(z)=$ $S\left(z I_{N}-L\right)^{-1} R$ is scalar strictly proper rational. The next result characterizes which outputs of the SISO interconnected system lead to a network without finite zeros, for given state and input interconnection matrices.

Theorem 4 (SISO Design Condition): Assume that $\left(A_{i}, b_{i}, c_{i}\right)$ are identical minimal SISO systems. Let $(L, R)$ be reachable with $L \in \mathbb{R}^{N \times N}, R \in \mathbb{R}^{N}$. Then a network output $S \in \mathbb{R}^{1 \times N}$ defines a minimal network realization $(\mathbf{A}, \mathbf{B}, \mathbf{C}, 0)$ without finite zeros if and only if $S\left(z I_{N}-L\right)^{-1} R$ has relative degree $N$.

Proof: By Corollary 1, the homogeneous network $(\mathbf{A}, \mathbf{B}, \mathbf{C}, 0)$ has no finite zeros if and only if this holds for $(L, R, S, 0)$. In the SISO case this is equivalent to the transfer function $S\left(z I_{N}-L\right)^{-1} R$ having no zeros. By [9], $(\mathbf{A}, \mathbf{B}, \mathbf{C}, 0)$ is minimal if and only if $(L, R, S)$ is minimal. In either case, $S\left(z I_{N}-L\right)^{-1} R$ has McMillan degree $N$ and has no zeros if and only if the relative degree of $S\left(z I_{N}-L\right)^{-1} R$ is equal to $N$. 


\section{Circulant Homogeneous Networks}

Homogeneous networks with special coupling structures appear in many applications, such as cyclic pursuit [14]; shortening flows in image processing [4] or the discretization of partial differential equations [3]. Here we characterize zeros for interconnections that have a circulant structure. A homogeneous network (2) is called circulant if the state-tostate coupling matrix $L$ is a circulant, i.e.

$$
\begin{aligned}
L & =\operatorname{Circ}\left(c_{0}, \ldots, c_{N-1}\right) \\
& =\left(\begin{array}{ccccc}
c_{0} & c_{1} & \ldots & c_{N-2} & c_{N-1} \\
c_{N-1} & c_{0} & c_{1} & \ldots & c_{N-2} \\
\vdots & \ddots & \ddots & \ddots & \vdots \\
c_{2} & \cdots & c_{N-1} & c_{0} & c_{1} \\
c_{1} & c_{2} & \cdots & c_{N-1} & c_{0}
\end{array}\right) .
\end{aligned}
$$

The book [7] provides algebraic background on circulant matrices. A basic fact on circulant matrices is that they are simultaneously diagonalizable by the Fourier matrix:

$$
\Phi=\frac{1}{\sqrt{N}}\left(\begin{array}{ccccc}
1 & 1 & 1 & \ldots & 1 \\
1 & \omega & \omega^{2} & \ldots & \omega^{N-1} \\
1 & \omega^{2} & \omega^{4} & \ldots & \omega^{2 N-2} \\
\vdots & & & & \\
1 & \omega^{N-1} & \omega^{2 N-2} & \ldots & \omega^{(N-1)^{2}}
\end{array}\right),
$$

where $\omega=e^{2 \pi j / N}$ denotes a primitive $N$-th root of unity. Note, that $\Phi$ is both a unitary and a symmetric matrix. It is then easily seen that any circulant matrix $L$ has the form $L=\Phi \operatorname{diag}\left(p_{L}(1), p_{L}(\omega), \ldots, p_{L}\left(\omega^{N-1}\right)\right) \Phi^{*}$, where $p_{L}(z):=\sum_{k=0}^{N-1} c_{k} z^{k-1}$. As a consequence of the preceding analysis we obtain

Theorem 5: Let $D$ be full rank and $M=$ $\operatorname{diag}\left(p_{L}(1), \ldots, p_{L}\left(\omega^{N-1}\right)\right)$ and $w_{1}, \ldots, w_{N}$ denote the complex roots of

$$
\operatorname{det}\left(\begin{array}{cc}
w I_{N}-M & \Phi^{*} R \\
S \Phi & D
\end{array}\right)=0 .
$$

Then

$$
\bigcup_{k=1}^{N}\left\{z \in \mathbb{C} \mid q(z)-w_{k} p(z)=0\right\}
$$

are the finite zeros of the homogeneous network $(\mathbf{A}, \mathbf{B}, \mathbf{C}, D)$.

\section{ZEROS FOR BLOCKED INTERCONNECTED Systems}

The well-known technique of blocking or lifting has been developed in systems and control [5] and signal processing [21]. In the systems and control literature, this method has mostly been exploited to transform linear discrete-time periodic systems to linear time-invariant systems so that the well-developed tools for linear time-invariant systems can be extended for design and analysis of linear discrete-time periodic systems; see [1] and the literature therein. Here we show how this technique can be applied to network control systems of the form

$$
\begin{aligned}
x(t+1) & =\mathbf{A} x(t)+\mathbf{B} u(t) \\
y(t) & =\mathbf{C} x(t)+D u(t),
\end{aligned}
$$

with matrices

$$
\mathbf{A}:=A+B L C \quad \mathbf{B}:=B R, \quad \mathbf{C}:=S C .
$$

and network transfer function

$$
\Gamma(z)=D+S C(z I-A-B L C)^{-1} B R .
$$

Here $x(t) \in \mathbb{R}^{\bar{n}}, y(t) \in \mathbb{R}^{p}$ and $u(t) \in \mathbb{R}^{m}$ and $A=\operatorname{diag}\left(A_{1}, \ldots, A_{N}\right), B=\operatorname{diag}\left(B_{1}, \ldots, B_{N}\right), C=$ $\operatorname{diag}\left(C_{1}, \ldots, C_{N}\right)$ are block-diagonal. Given an integer $T \geq$ 1 as the block size, we define for $t=0, T, 2 T, \ldots$

$$
\begin{aligned}
& U(t)=\left(\begin{array}{llll}
u(t)^{\top} & u(t+1)^{\top} & \ldots & u(t+T-1)^{\top}
\end{array}\right)^{\top}, \\
& Y(t)=\left(\begin{array}{llll}
y(t)^{\top} & y(t+1)^{\top} & \ldots & y(t+T-1)^{\top}
\end{array}\right)^{\top} .
\end{aligned}
$$

The blocked system then is defined as [1]:

$$
\begin{aligned}
x(t+T) & =\mathbf{A}_{b} x(t)+\mathbf{B}_{b} U(t), \\
Y(t) & =\mathbf{C}_{b} x(t)+\mathbf{D}_{b} U(t),
\end{aligned}
$$

where

$$
\begin{aligned}
\mathbf{A}_{b} & =\mathbf{A}^{T}, \quad \mathbf{B}_{b}=\left(\begin{array}{llll}
\mathbf{A}^{T-1} \mathbf{B} & \mathbf{A}^{T-2} \mathbf{B} & \ldots & \mathbf{B}
\end{array}\right), \\
\mathbf{C}_{b} & =\left(\begin{array}{ccccc}
\mathbf{C}^{\top} & \mathbf{A}^{\top} \mathbf{C}^{\top} & \ldots & \mathbf{A}^{(T-1)^{\top}} \mathbf{C}^{\top}
\end{array}\right)^{\top}, \\
\mathbf{D}_{b} & =\left(\begin{array}{cccc}
D & 0 & \ldots & 0 \\
\mathbf{C B} & D & \ldots & 0 \\
\vdots & \vdots & \ddots & \vdots \\
\mathbf{C A}^{T-2} \mathbf{B} & \mathbf{C A}^{T-3} \mathbf{B} & \ldots & D
\end{array}\right)
\end{aligned}
$$

The transfer function $\Gamma_{b}\left(z^{T}\right)=\mathbf{D}_{b}+\mathbf{C}_{b}\left(z^{T} I-\mathbf{A}_{b}\right)^{-1} \mathbf{B}_{b}$ of (15), see [1], [13], has the circulant-like structure as

$$
\left(\begin{array}{ccccc}
H_{0}(z) & H_{T-1}(z) & \ldots & H_{2}(z) & H_{1}(z) \\
z H_{1}(z) & H_{0}(z) & H_{T-1}(z) & \ldots & H_{2}(z) \\
\vdots & \ddots & \ddots & \ddots & \vdots \\
z H_{T-2}(z) & \ldots & z H_{1}(z) & H_{0}(z) & H_{T-1}(z) \\
z H_{T-1}(z) & z H_{T-2}(z) & \ldots & z H_{1}(z) & H_{0}(z)
\end{array}\right)
$$

where $H_{0}(z)=D+\mathbf{C}\left(z I-\mathbf{A}^{T}\right)^{-1} \mathbf{A}^{T-1} \mathbf{B}$ and $H_{k}(z)=$ $\mathbf{C}\left(z I-\mathbf{A}^{T}\right)^{-1} \mathbf{A}^{k-1} \mathbf{B}, k=1, \ldots, T-1$. It is worthwhile pointing out that the blocked transfer function has the structure of a generalized circulant matrix. The theory of generalized circulant matrices is very similar to that of classical circulant matrices; see [7]. Using such techniques we obtain the following result.

Proposition 1: Let $\Phi$ denote the Fourier matrix and $\Gamma(z)=P(z) Q(z)^{-1}$ a right coprime factorization of the network transfer function.

1) For all nonzero complex numbers $z \in \mathbb{C}$, the transfer function of the blocked system is

$$
\Gamma_{b}\left(z^{T}\right)=L_{\Gamma}(z)\left(\begin{array}{cccc}
\Gamma(z) & & & 0 \\
& \Gamma(\omega z) & & \\
& & \ddots & \\
& & & \Gamma\left(\omega^{T-1} z\right)
\end{array}\right) R_{\Gamma}(z)
$$

where

$$
\begin{aligned}
& L_{\Gamma}(z)=\operatorname{diag}\left(1, z, \ldots, z^{T-1}\right) \Phi \otimes I_{p} \\
& R_{\Gamma}(z)=\operatorname{diag}\left(1, z, \ldots, z^{T-1}\right) \Phi \otimes I_{m}
\end{aligned}
$$


are invertible for $z \neq 0$. Moreover, we have the factorization

$$
\Gamma_{b}\left(z^{T}\right)=P_{b}(z) Q_{b}(z)^{-1}
$$

which is coprime for $z \neq 0$. Here

$$
\begin{aligned}
& P_{b}(z)=L_{\Gamma}(z) \operatorname{diag}\left(P(z), \ldots, P\left(\omega^{T-1} z\right)\right) \\
& Q_{b}(z)=R_{\Gamma}(z) \operatorname{diag}\left(Q(z), \ldots, Q\left(\omega^{T-1} z\right)\right) .
\end{aligned}
$$

2) Consider the system matrices

$$
\begin{aligned}
\Sigma_{b}(z) & =\left(\begin{array}{cc}
z I_{\bar{n}}-\mathbf{A}_{b} & -\mathbf{B}_{b} \\
\mathbf{C}_{b} & \mathbf{D}_{b}
\end{array}\right), \\
\hat{\Sigma}_{b}(z) & =\left(\begin{array}{cc}
I_{\bar{n}(T-1)} & 0 \\
0 & \Sigma_{b}(z)
\end{array}\right) .
\end{aligned}
$$

There exist polynomial matrices $L(z)$ and $R(z)$, that are invertible for all nonzero complex numbers $z \in \mathbb{C}$, such that

$$
\begin{aligned}
& \hat{\Sigma}_{b}\left(z^{T}\right)=
\end{aligned}
$$

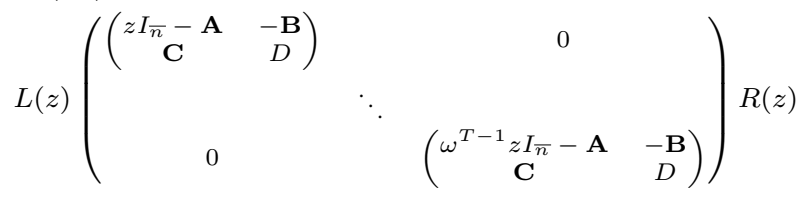

The preceding results lead to the following conclusion concerning finite zeros of interconnected systems. Thus consider the interconnected system $(\mathbf{A}, \mathbf{B}, \mathbf{C}, D)$ defined in (2). Let $\left(\mathbf{A}_{\mathbf{b}}, \mathbf{B}_{\mathbf{b}}, \mathbf{C}_{\mathbf{b}}, D_{b}\right)$ denote the associated blocked system. The proof of the next result uses techniques from [22]. We omit the details.

Theorem 6: 1) A complex number $Z \neq 0$ is a finite zero of the blocked network $\left(\mathbf{A}_{\mathbf{b}}, \mathbf{B}_{\mathbf{b}}, \mathbf{C}_{\mathbf{b}}, D_{b}\right)$ if and only if there exists $z \in \mathbb{C}$ with $z^{T}=Z$ such that $z$ is a finite zero of $(\mathbf{A}, \mathbf{B}, \mathbf{C}, D)$.

2) Let $D$ be full rank and $(\mathbf{A}, \mathbf{B}, \mathbf{C}, D)$ a homogeneous network. Then the blocked network $\left(\mathbf{A}_{\mathbf{b}}, \mathbf{B}_{\mathbf{b}}, \mathbf{C}_{\mathbf{b}}, D_{b}\right)$ has no zeros at infinity. The finite zeros of $\left(\mathbf{A}_{\mathbf{b}}, \mathbf{B}_{\mathbf{b}}, \mathbf{C}_{\mathbf{b}}, D_{b}\right)$ are exactly all $Z=z^{T}$ such that $h\left(\omega^{k} z\right)$ is a finite zero of $(L, R, S, D)$ for some $0 \leq$ $k \leq T-1$.

\section{CONCLUSions}

In this paper, we study the zero properties of networks of linear multi-agent systems. It is assumed that the parameters defining the interconnection topology are constant. The zeros are characterized for both homogenous and heterogenous networks. In particular, it is shown that for homogenous networks with a direct feedthrough matrix of full rank, then the finite zeros of the whole network are exactly the preimages of zeros by inverse of an agent transfer function. We then discussed the condition under which networks of multi-agent systems have no finite nonzero zeros. The tool of generalized circulant matrices is introduced for a concise analysis of zeros of blocked network systems. In future work we will address open problems such as the consideration of periodically varying network topologies and MIMO dynamics for each agents.

\section{REFERENCES}

[1] S. Bittanti and P. Colaneri, Periodic Systems Filtering and Control, ser. Communications and Control Engineering. Springer-Verlag, 2009.

[2] P. Bolzern, P. Colaneri, and R. Scattolini, "Zeros of discrete-time linear periodic systems," IEEE Transactions on Automatic Control, vol. 31, no. 11 , pp. 1057 - 1058, Nov. 1986

[3] R. W. Brockett and J. L. Willems, "Discretized partial differential equations: Examples of control systems defined on modules," Automatica, vol. 10, no. 5, pp. 507 - 515, 1974.

[4] A. M. Bruckstein, G. Sapiro, and D. Shaked, "Evolutions of planar polygons." International Journal of Pattern Recognition and Artificial Intelligence, vol. 9, no. 6, pp. 991-1014, 1995.

[5] T. Chen and B. A. Francis, Optimal Sampled-Data Control Systems. Secaucus, NJ, USA: Springer-Verlag New York, Inc., 1995.

[6] W. Chen, B. D. Anderson, M. Deistler, and A. Filler, "Properties of blocked linear systems," Automatica, vol. 48, no. 10, pp. 2520 - 2525, 2012.

[7] P. J. Davis, Circulant matrices. John Wiley and Sons. New York, 1979.

[8] J. A. Fax and R. M. Murray, "Information flow and cooperative control of vehicle formations," IEEE Transactions on Automatic Control, vol. 49, no. 9, pp. 1465-1476, 2004.

[9] P. A. Fuhrmann and U. Helmke, "Observability and strict equivalence of networked linear systems," Mathematics of Control, Signals, and Systems, 2013.

[10] P. A. Fuhrmann, "On strict system equivalence and similarity," International Journal of Control, vol. 25, no. 1, pp. 5-10, 1977.

[11] O. M. Grasselli and S. Longhi, "Zeros and poles of linear periodic multivariable discrete-time systems," Circuits, Systems, and Signal Processing, vol. 7, pp. 361-380, 1988.

[12] T. Kailath, Linear Systems. Prentice-Hall, New Jersey, 1980.

[13] P. Khargonekar, K. Poolla, and A. Tannenbaum, "Robust control of linear time-invariant plants using periodic compensation," IEEE Transactions on Automatic Control, vol. 30, no. 11, pp. 1088 - 1096, Nov. 1985.

[14] J. Marshall, M. Broucke, and B. Francis, "Formations of vehicles in cyclic pursuit," IEEE Transactions on Automatic Control, vol. 49, no. 11 , pp. 1963 - 1974, nov. 2004.

[15] Y. Mo, T.-H. Kim, K. Brancik, D. Dickinson, H. Lee, A. Perrig, and B. Sinopoli, "Cyber-physical security of a smart grid infrastructure," Proceedings of the IEEE, vol. 100, no. 1, pp. 195-209, 2012.

[16] R. Olfati-Saber, J. A. Fax, and R. M. Murray, "Consensus and cooperation in networked multi-agent systems," Proceedings of the IEEE, vol. 95, no. 1, pp. 215-233, 2007.

[17] W. Ren, R. Beard, and E. Atkins, "Information consensus in multivehicle cooperative control," IEEE Control Systems Magazine, vol. 27, no. 2 , pp. $71-82$, april 2007.

[18] H. H. Rosenbrock, State-Space and Multivariable Theory. London, UK: Nelson, 1970.

[19] — Computer-Aided Design of Control Systems. Cambridge, London, 1974.

[20] A. Teixeira, I. Shames, H. Sandberg, and K. Johansson, "Revealing stealthy attacks in control systems," in Communication, Control, and Computing (Allerton), 2012 50th Annual Allerton Conference on, 2012, pp. 1806-1813.

[21] P. P. Vaidyanathan, Multirate Systems and Filter Banks. Upper Saddle River, NJ, USA: Prentice-Hall, Inc., 1993.

[22] M. Zamani, B. D. O. Anderson, U. Helmke, and W. Chen, "On the zeros of blocked time-invariant systems," submitted for publication.

[23] M. Zamani, W. Chen, B. D. O. Anderson, M. Deistler, and A. Filler, "On the zeros of blocked linear systems with single and mixed frequency data," Proceedings of the 2011 Control and Desicion Conference, pp. 4312-4317, 2011. 